\title{
Fluctuations and Their Correlations in Econophysics
}

\author{
Y. Liu, L.A.N. Amaral, P. Cizeau, P. Gopikrishnan, \\ M. Meyer, C.-K. Peng and H.E. Stanley \\ Center for Polymer Studies and Department of Physics \\ Boston University, Boston, MA 02215, USA
}

\begin{abstract}
We address a current question in econophysics: Are fluctuations in economic indices correlated? To this end, we analyze 1-minute data on a stock index, the Standard and Poor index of the 500 largest stocks. We extend the 6-year data base studied by Mantegna and Stanley by including the 13 years 1984-1996 inclusive, with a recording frequency of 15 seconds. The total number of data points in this 13 years period exceed 4.5 million, which allows for a very detailed statistical analysis. We find that the fluctuations in the volatility are correlated, and that the correlations are well described by a power law.
\end{abstract}

\section{Introduction}

Today we are going to look at some examples of scale-invariant correlations that are of interest to social scientists.

At one time, it was imagined that the "scale-free" phenomena are relevant to only a fairly narrow slice of physical phenomena (Stanley 1971). However, the range of systems that apparently display power law and hence scale-invariant correlations has increased dramatically in recent years, ranging from base pair correlations in noncoding DNA (Peng et al. 1992, Arneodo et al. 1995), lung inflation (Suki et al. 1994, Barabási et al. 1996) and interbeat intervals of the human heart (Peng et al. 1993, Peng et al. 1995, Ivanov et al. 1996, Ivanov et al. 1998) to complex systems involving large numbers of interacting subunits that display "free will," such as city growth (Makse et al. 1995, Makse et al. preprint, Zanette and Manrubia 1997, Ball 1998), animal behaviour (Peterson 1996, Peterson 1997, Viswanathan et al. 1996, Viswanathan et al. submitted, Keitt and Stanley 1998, Keitt and Stanley, preprint), and even economics (Mandelbrot 1997, Mantegna et al. 1997). In particular, economic time series, as e.g., stock market indices or currency exchange rates depend on the evolution of a large number of strongly interacting systems far from equilibrium, and belong to the class of a complex evolving systems. Thus, the statistical properties of financial markets have attracted the interests of many physicists (Bak et al. 1994, Stanley et al. 1996, Levy et al. 1994; Levy et al. 1995, Bouchaud et al. 1994; Sornette et al. 1996, Mantegna and Stanley 1995; 1994, Ghashghaie et al. 1996; Mantegna and Stanley 1996; Arneodo et al. preprint, Levy et al. 1996, Potters et al. 
preprint, Takayayasu et al. 1992, Hirabayashi et al. 1993, Takayayasu and Okuyama 1998, Krugman 1996). Methods originating in statistical physics have been proven useful in analyzing financial indices. They are also used to construct new models for the pricing of derivatives and the assessment of the involved risk (Bouchaud et al. 1994; Sornette et al. 1996).

The recent availability of very high frequency data allows to study economic time series with a high accuracy on a wide range of time scales varying from less than 1 minute up to more than 10 years. Consequently, a large number of methods known from statistical physics have been applied to characterize the time evolution of stock prices and foreign exchange rates (Bak et al. 1994, Stanley et al. 1996, Levy et al. 1994; Levy et al. 1995, Bouchaud et al. 1994; Sornette et al. 1996, Mantegna and Stanley 1995; 1994, Takayayasu et al. 1992, Hirabayashi et al. 1993, Takayayasu and Okuyama 1998, Krugman 1996). It turns out that the distributions of the increments of economic time series, both in stock market indices and foreign currency exchange rates, are nearly symmetric and have strong "leptokurtic" wings (Mandelbrot 1963, Mantegna and Stanley 1995; 1994). Index increments as a function of time show only weak correlations on short time scales below 10 minutes (Mandelbrot 1963, Fama 1970), which seemingly makes them fundamentally different from well known examples of complex dynamic systems in physics such as, e.g., turbulent flow where power law correlations on long time scales are commonly observed (Kolmogorov 1961).

The situation is different for the volatility, i.e., the market fluctuations averaged on a suitable time interval. There is long time persistence much larger than the correlation time in volatility (Ding et al. 1983). Volatility is the key input of virtually all option pricing models, including the classic Black and Scholes (Black and Scholes 1973) and Cox, Ross, and Rubinstein (Cox et al. 1979) binomial models that are based on estimates of the asset's volatility over the remaining life of the option. So to understand the dynamics of the volatility has very important practical reason.

Here, we quantify long range power law correlations in the volatility of the S\&P 500 stock index and report an occurrence of a cross-over phenomena of this long range correlation. Furthermore, we discuss the distribution of the volatility, and show that it can be fitted very well by a log-normal distribution.

\section{Quantification of Correlations in S\&P 500}

\subsection{Data Description and Detrending}

The S\&P 500 index, an index of the New York Stock Exchange, consists of the 500 largest companies in the US. It is a market-value weighted index (stock price times number of shares outstanding), with each stock's weight in the index proportionate to its market value. The S\&P 500 index is one of the most widely used benchmarks of U.S. equity performance. Our data cover 13 years (from Jan 1984 to Dec 1996) with a recording frequency of 15 seconds 
interval. The total number of data points in this 13 years period exceed 4.5 million, which allows for a very detailed statistical analysis.

The S\&P 500 index $Z(t)$ from 1984 to 1996 tends to increase constantly on a semi-log graph except during crashes, e.g., October 1987 and May 1990. Since the standard deviation of $Z(t+\Delta t)-Z(t)$ is proportional to the price level, we take the logarithmic of the index as everyone does. We define the forward change

$$
G(t) \equiv \log _{e} Z(t+\Delta t)-\log _{e} Z(t),
$$

where $\Delta t$ is the time-lag (set to 1 minute in the correlation study).

We only count the number of minutes during the opening hours of the stock market, and remove the nights, weekends and holidays from the data set, i.e., the closing and the next opening of the market is continuous.

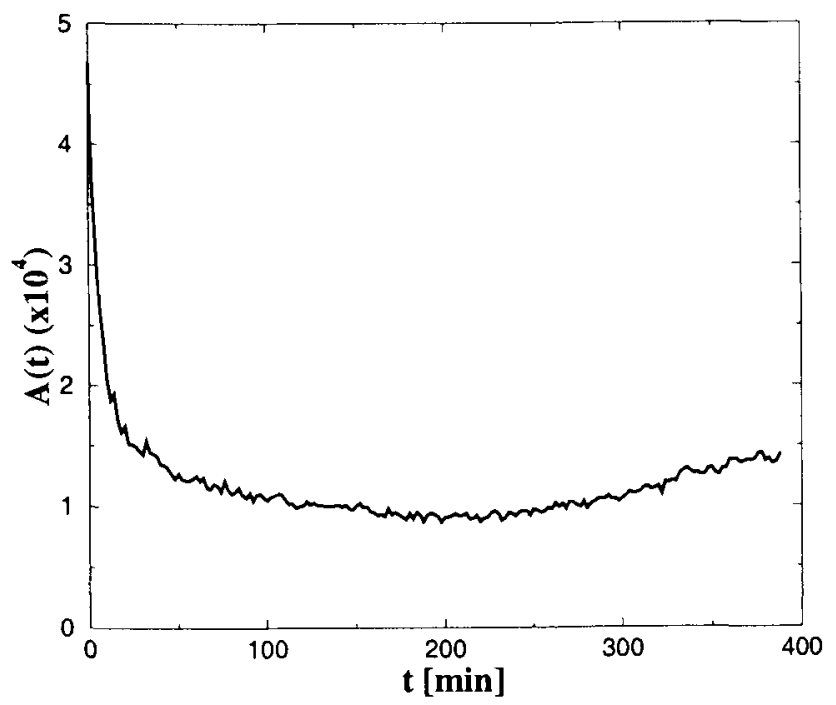

Fig. 1. The intra-day pattern $I(t)$ of S\&P 500 index marked by 1 -minute interval. Before Sept. 30, 1985, the data length is 6 hours per day. After then, it's $6 \frac{1}{2}$ hours. Two curves have the same pattern, only the latter curve is shown here. Big changes in the price happens within the first 10 minutes when the market opens.

The absolute value of $G(t)$ describes the amplitude of the fluctuation. $|G(t)|$ is, by definition, always positive, and there are no obvious global trends visible, which is due to the logarithmic difference, i.e. the relative increment on the original index $Z(t)$. The large values of $Z(t)$ correspond to the crashes and big rallies of the index. It is known in the financial literature that the volatility varies in time (Bollerslev et al. 1992; Schert 1989; Gallant et al. 
1992; Le Baron 1992; Chan et al. 1991), as expected the $|G(t)|$ quantity also fluctuates in time.

It is known that there exits intra-day patterns in NYSE and S\&P 500 index data, one simple explanation is that there are many information traders active near the open and many liquidity traders active near the close (Admati and Pfleiderer 1988a). We find the similar intra-day pattern in our S\&P 500 index data set [Fig. (1)]. The intra-day pattern

$$
A(t) \equiv \frac{\sum_{i=1}^{N}\left|G\left(t_{i, \text { same }}\right)\right|}{N},
$$

where $N$ is the total trading days over the 13-year period and $t_{\text {same }}$ is the same time of each day ( $N=3309$ in our study). In order to avoid the artificial correlation caused by this daily oscillation, $G(t)$ signal is normalized by the intra-day pattern

$$
g(t) \equiv G(t) / A(t),
$$

i.e. each data point divided by the intra-day pattern of its corresponding time during the day.

\subsection{Methods to Calculate Correlations}

We have three methods to quantify the correlations. The direct method to study the correlation property is the correlation function estimation, which is defined as

$$
C(\tau) \equiv \frac{\langle G(t) G(t+\tau)\rangle-\langle G(t)\rangle^{2}}{\left\langle G^{2}(t)\right\rangle-\langle G(t)\rangle^{2}},
$$

where $\tau$ is the time lag. The problem with the correlation function estimation is that it depends on the estimated average value of the time series. Since it is difficult to calculate the true average value, the correlation function can only give us a qualitative estimation (Beran 1994).

Another method to calculate the correlation functions is the traditional power spectrum analysis. Since this method can only apply to linear and stationary (or strictly periodic) time series, although it could give quantitative measures, we still need other method to confirm its results.

We applied the third method-termed detrended fluctuation analysis (DFA) (Peng et al. 1994, Peng et al. 1995) - to quantify the correlation exponent. The advantages of DFA over conventional methods (e.g. spectral analysis and Hurst analysis) are that it permits the detection of long-range correlations embedded in a nonstationary time series, and also avoids the spurious detection of apparent long-range correlations that are an artifact of nonstationarities. This method has been validated on control time series that consist of long-range correlations with the superposition of a nonstationary external trend (Peng et al. 1994). The DFA method has also been successfully 


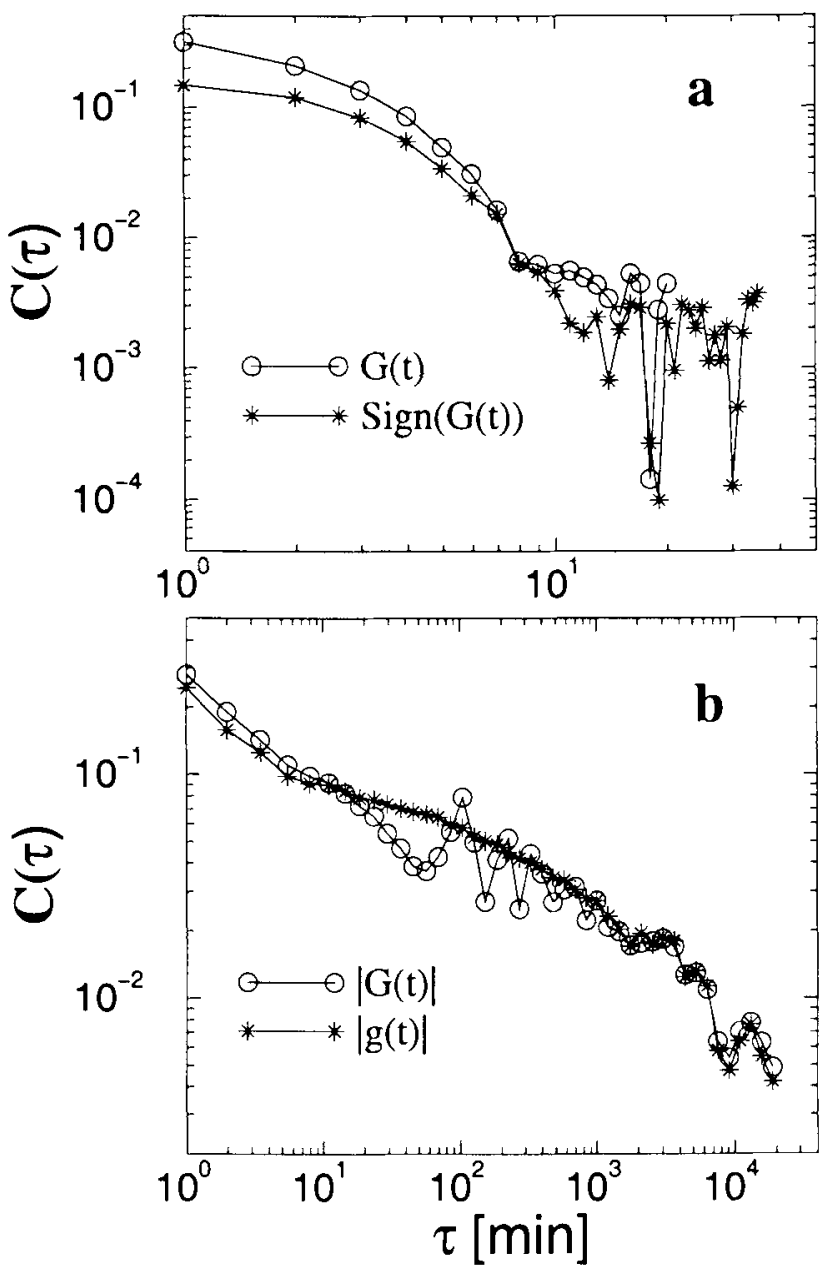

Fig. 2. (a) Semi-log plot of correlation functions of $g(t)$ and $|g(t)|$, (b) The same correlations in the double log plot. The $g(t)$ decays exponentially to 0 within half an hour. But the curve of $|g(t)|$ decays very slowly for more than 4 decades. A power law correlation seems to exist in the $|g(t)|$. Note that both graphs are truncated at the first zero value of $C(\tau)$.

applied to detect long-range correlations in highly complex heart beat time series (Peng et al. 1995, Iyengar et al. 1997), and other physiological signals (Hausdorff et al. 1996, Hausdorff and Peng 1996).

A detailed description of the DFA algorithm appears elsewhere (Peng et al. 1994, Peng et al. 1995). Briefly, the $|g(t)|$ time series (with $N$ data) is first integrated, 


$$
y(t) \equiv \sum_{i=1}^{t}|g(i)|
$$

Next the integrated time series is divided into boxes of equal length, $n$. In each box of length $n$, a least squares line is fit to the data (representing the trend in that box). The $y$ coordinate of the straight line segments is denoted by $y_{n}(t)$. Next we detrend the integrated time series, $y(t)$, by subtracting the local trend, $y_{n}(t)$, in each box. The root-mean-square fluctuation of this integrated and detrended time series is calculated by

$$
F(n)=\sqrt{\frac{1}{N} \sum_{t=1}^{N}\left[y(t)-y_{n}(t)\right]^{2}} .
$$

This computation is repeated over all time scales (box sizes) to provide a relationship between $F(n)$, the average fluctuation as a function of box size. In our case, the box size $n$ ranged from $10 \mathrm{~min}$ to $10^{5} \mathrm{~min}$, the upper bound of $n$ is determined by the actual data length. Typically, $F(n)$ will increase with box size $n$. A linear relationship on a double log graph indicates the presence of power law (fractal) scaling. Under such conditions, the fluctuations can be characterized by a scaling exponent $\alpha$, the slope of the line relating $\log F(n)$ to $\log n$.

For exactly self-similar process, as e.g. fractional Brownian motion, the DFA exponent $\alpha$ is related to the power spectrum exponent $\beta$ through the relation $\alpha=(1+\beta) / 2$ (Beran 1994). The calculation of $F(n)$ can distinguish four types of behavior. (1) Uncorrelated time series give rise to uncorrelated random walks described by $F(n) \sim n^{\alpha}$ with $\alpha=1 / 2$, as expected from the central limit theorem. Power spectrum would be flat with $\beta=0$. (2) Markov processes with a characteristic correlation length $t_{0}$, gives $C(\tau) \sim \exp \left(-\tau / t_{0}\right)$; For $t<t_{0}$, it is the Brownian process with $\alpha=1.5$ and corresponding $\beta=2$, nonetheless the asymptotic behavior for sufficiently large $t$ with $\alpha=1 / 2$ would be unchanged from the purely random case. (3) In the presence of long-range correlations with no characteristic time scale, the scaling property would be a power law function with $\alpha \neq 1 / 2$ and $\beta \neq 0$.

\subsection{Correlation Results of S\&P 500 Index}

Using correlation function estimation, we find that the correlation function of $g(t)$ decays exponentially with a characteristic time of the order of 1 $10 \mathrm{~min}$, but the absolute value $|g(t)|$ does not (Fig. 2). This result is consistent with previous studies on several economic series (Fama 1970, Ding et al. 1983, Dacorogna et al. 1993).

The power spectrum calculation of $|g(t)|$ shows that the data fit not one but rather two separate power laws: for $f>f_{\times}$the power law exponent is $\beta_{1}=0.31$, while for $f<f_{\times}$the exponent $\beta_{2}=0.90$ is three times larger; here $f_{\times}$is called the crossover frequency. 
DFA method confirms our power spectrum results. From the behavior of the power spectrum, we expect that the DFA method will also predict two distinct regions of power law behavior, with exponents $\alpha_{1}=0.66$ and $\alpha_{2}=0.95$ for $t$ less than or greater than a characteristic time scale $t_{\times} \equiv 1 / f_{\times}$, where we have used the relation

$$
\alpha=(1+\beta) / 2
$$

The data yield $\alpha_{1}=0.66, \alpha_{2}=0.93$, thereby confirming the consistency of the power spectrum and DFA methods. Also the crossover time is very close to the result obtained from the power spectrum, with

$$
t_{\times} \approx 1 / f_{\times} \approx 600 \min
$$

about 1.5 trading days.

To test whether this correlation is due to the distribution function, we shuffled each point of the $|g(t)|$ time series randomly. The shuffling operation keeps the distribution of $|g(t)|$ unchanged, but kills the correlations in the time series totally if there are any. DFA measurement of this randomly shuffled data does not show any correlations and gives exponent $\alpha=0.50$. This tells us that the long-range correlations are actually due to the dynamics of the economic system and not simple due to the distribution.

The observed long range correlation and the crossover behavior noted above is from the entire 13-year period, so it is natural to enquire whether it will still hold for periods smaller than 13 years. Therefore, we choose a sliding window (with size 1 year) and calculate both exponents $\alpha_{1}$ and $\alpha_{2}$ within this window as the window is dragged, down the data set with one month step. We find that the value of $\alpha_{1}$ is very "stable" (independent of the position of the window) fluctuating around the mean value $2 / 3$. Surprisingly, however, the variation of $\alpha_{2}$ is much greater, showing sudden jumps when very volatile periods enter or leave the time window.

We studied several standard mathematical models, such as fractional Brownian motion (Beran 1994, Mandelbrot and van Ness 1968) and fractional ARIMA processes (Granger and Ding 1996), commonly used to account for long-range correlation in a time series and found that none of them can reproduce the large fluctuation of $\alpha_{2}$.

\section{The Volatility Distribution of S\&P 500}

The volatility is a measure of the mean fluctuation of a market price over a certain time interval $T$. The volatility is of practical importance since it quantifies the risk related to assets (Bouchaud et al. 1994; Sornette et al. 1996). As shown above, unlike price changes that are correlated only on very short time scales (Fama 1970) (a few minutes), the absolute values of price changes (which are closely related to the volatility) show correlations on time 
scales up to many years (Ding et al. 1983, Dacorogna et al. 1993, Liu et al. 1997, Cizeau et al. 1997).

The same data set of the S\&P 500 index of the New York stock exchange is explored here to study the volatility distribution. This data set has been extended by 7 years the data set previously analyzed in (Mantegna and Stanley $1995 ; 1994)$.

We calculate the logarithmic increments $G(t)$ in Eq. 1, where $G(t)$ is the relative price change $\Delta Z / Z$ in the limit $\Delta t \rightarrow 0$. Here we set $\Delta t=30 \mathrm{~min}$, well above the correlation time of the price increments; and we obtain similar results for other choices of $\Delta t$ (larger than the correlation time).

As we show in the correlation discussion, there is a strong "U-shape" market activity over the day. To remove artificial correlations resulting from this intra-day pattern of the volatility (Wood et al. 1985, Harris 1986, Admati and Pfleiderer 1988a, Ekman 1992), we normalized $|G(t)|$ by $A(t)$ as shown in Eq. 3.

We obtain the volatility at a given time by averaging $|g(t)|$ over a time window $T=n \cdot \Delta t$ with some integer $n$,

$$
v_{T}(t) \equiv \frac{1}{n} \sum_{t^{\prime}=t}^{t+n-1}\left|g\left(t^{\prime}\right)\right|
$$

The volatility fluctuates strongly showing a marked maximum for the 1987 crash. Generally periods of high volatility are not independent but tend to "cluster." This clustering is especially marked around the 1987 crash, which is accompanied by precursors (possibly related to oscillatory patterns (Bouchaud et al. 1994; Sornette et al. 1996)). Clustering occurs also at other times (e.g., during the second half of 1990), while there are extended periods where the volatility remains at a rather low level (e.g., in 1985 and 1993).

When we consider a scaled probability distribution $P\left(v_{T}\right)$ for several values of $T$, the data for different averaging windows collapse to one curve. Remarkably, the scaling form is log-normal, not Gaussian. In the limit of very long averaging times, one expects that $P\left(v_{T}\right)$ becomes Gaussian, since the central limit theorem holds also for correlated series (Beran 1994), with a slower convergence than for non-correlated processes (Potters et al. preprint, Cont 1977). However, a log-normal fits the data better than a Gaussian.

Correlations can be accurately quantified using detrended fluctuation analysis (Peng et al. 1994). The analysis reveals power-law behavior independent of the $T$ value chosen with an exponent $\alpha \cong 0.9$ in agreement with the value found for the absolute price increments (see Sec. 2).

\section{Discussion of Empirical Results on Finance}

In this study, we have used the DFA method to display correlation in the volatility of S\&P 500 index. We find that the volatility is highly correlated, 
and that the correlation is remarkably long range, indeed, over 5 decades. Moreover, the quantitative scaling of the correlation follows the power law form observed in numerous phenomena which have a self-similar or "fractal" origin.

We have also found that the probability distribution of the S\&P 500 volatility can be well described by a log-normal function. This functional shape does not depend on the averaging time interval $T$ used to calculate volatility $v_{T}(t)$. The log-normal shape of the distribution is consistent with a multiplicative process (Bunde and Havlin 1996) for the volatility (Ghashghaie et al. 1996; Mantegna and Stanley 1996; Arneodo et al. preprint). However, a multiplicative behavior would be surprising, because efficient market theories (Fama 1970) assume that the price reflects all current information that could anticipate future events and the price changes, $G(t)$, are caused by incoming new informations about an asset. Since such information-induced price changes are additive in $G(t)$, they should not give rise to multiplicative behavior of the volatility.

To account for the time dependence of the volatility and its long-range correlations, ARCH (Engle 1982), GARCH (Bollerslev 1986) models and related approaches (Granger and Ding 1996) have been developed, which assume that the volatility depends on time and on the past evolution of the index. It may be also worthwhile to test these models with regard to the volatility distribution $P\left(v_{T}\right)$.

\section{Scale Invariance in Economics}

Economics is different than finance, and we have also looked at economic data. Specifically, in collaboration with a card-carrying economist, Michael Salinger--we studied the possibility that all the companies in a given economy might interact, more or less, like an Edwards-Anderson spin glass. As in an Edwards-Anderson spin glass, each spin interacts with another spin--but not with the same coupling and not even with the same sign.

If the sales in a given company $x$ decreases by, e.g., $10 \%$, it will have repercussions in the economy. Some of the repercussions will be favorablecompany $y$, which competes with $x$, may experience an increase in market share. Others will be negative- service industries that provide personal services for company $x$ employees may experience a drop-off in sales as employee salaries will surely decline. There are positive and negative correlations for almost any economic change. Can we view the economy as a complicated Ising system or spin glass?

To approach this interesting bit of statistical "poetry" and make sense of it, we first located and secured a database that lists the actual size of every firm in the United States. With this information, we did an analysis to determine how the distribution of firm size changes from one year to the next. We then made a histogram for each of three characteristic firm sizes. 
The largest firms have a very narrow distribution-plausible because the percentage of size change from year to year for the largest firms cannot be that great. On the other hand, a tiny company or a garage-based start-up can radically increase (or decrease) in size from year to year. The histograms have a width determined by the size of the firm. When this width is plotted on the $y$ axis of log-log paper as a function of the size of the firm on the $x$ axis, the data are approximately linear over 8 orders of magnitude, from the tiniest firms in the database to the largest. The width scales as the firm size to an exponent $\beta$, with $\beta \approx 1 / 6$ (Buldyrev et al. 1997a). We can therefore normalize the growth rate and show that all the data collapse on a single curve-demonstrating the scaling of this measure of firm size.

Why does this occur? We're working on that. If we model this firm structure as an approximate Cayley tree, in which each subunit of a firm reacts to its directives from above with a certain probability distribution. This model, developed primarily by Sergey Buldryev, seems to be consistent with the critical exponent $-1 / 6$ (Buldyrev et al. 1997b). More recently, Amaral et al. (Amaral et al. 1998) have proposed a microscopic model, and Takayasu (Takayayasu and Okuyama 1998) has extended the empirical results to a wide range of countries.

\section{Conclusions}

Is the point of this talk just to show that a lot of different systems appear to develop scale-invariant correlations? If so, how do we understand this empirical fact?

Bak's idea that systems self-organize themselves such that they are in effect near a critical point is an appealing unifying principle. Near a critical point, there is a delicate balance between the exponentially-growing number of different one-dimensional paths connecting any two faraway subunits and the exponentially-decaying correlations along each one-dimensional path (this concept is illustrated, e.g., in Fig. 9.4 of Ref. [1]. If the control parameter (say coupling constant) is too small, the correlations die out so fast along each one-dimensional path that subunits far from one another are not well correlated. However, at a critical point, the exponentially-large number of paths connecting each pair of subunits is sufficient to balance out the exponential decay along each path and the "correction factor" wins out-this correction factor is the power law that governs the total number of one-dimensional paths connecting two distant subunits. The exponent in this correction factor depends primarily on the system dimension, and not at all on the actual arrangement of the subunits (lattice or no-lattice).

Could it be that somehow social systems push themselves up "up to the limit"-just as a sandpile is pushed to the limit before an avalanche starts, an image that has attracted recent attention in the debate between "selforganized criticality" and "plain old criticality" (see, e.g., Vespignani and 
Zapperi (Vespignani and Zapperi 1997) and references therein)? For example, in economics every subunit plays according to rules and pushes itself up against the limits imposed by these rules. But social systems display a variety of rich forms of "order", far richer than we anticipate from studies of ferromagnets and antiferromagnets (see, e.g., some of the papers appearing in Knobler et al. (Knobler et al. 1997). Could such orderings arise from the complex nature of the interactions? Or from the range of different "sizes" of the constituent subunits as, e.g., one finds ordering in sandpiles when sand particles of two different grain sizes are dropped onto a heap-see, e.g., Refs. [66-69]. These are questions that occupy us now, and questions I would be delighted to discuss with any of the conference participants.

\section{Acknowledgments}

We thank S. Havlin, R. Mantegna, and S. Zapperi for very helpful discussions through the course of this work, and DFG, NIH, and NSF for financial support.

\section{References}

Admati, A., Pfleiderer, P. (1988): Review of Financial Studies 1, 3 Review of Financial

Amaral, L.A.N., Buldyrev, S.V., Havlin, S., Salinger, M.A., Stanley, H.E. (1998): Phys. Rev. Lett. 80, 1385

Arneodo, A., Bacry, E., Graves, P.V., Mugy, J.F. (1995): Phys. Rev. Lett. 74, 3293 Bak, P., Chen, K., Scheinkman, J.A., Woodford, M. (1993): Richerche Economichi 47, 3; Scheinkman, J.A., Woodford, J. (1994): American Economic Review 84, 417

Ball, P. (1998): The Self-Made Tapestry: Pattern Formation in Nature (Oxford University Press, Oxford)

Barabási, A.-L., Buldyrev, S.V., Stanley, H.E., Suki, B. (1996): Phys. Rev. Lett. 76, 2192

Beran, J. (1994): Statistics for Long-Memory Processes (Chapman \& Hall, NY)

Black, F., Scholes, M. (1973): J. of Political Economy 81, 637

Bollerslev, T. (1986): J. Econometrics 31, 307

Bollerslev, T., Chou, R.Y., Kroner, K.F. (1992): J. Econometrics 52, 5; Schwert, G.W. (1989): The Journal of Finance 44, 1115; Gallant, A.R., Rossi, P.E., Tauchen, G. (1992): The Review of Financial Studies 5, 199; Le Baron, B. (1992): Journal of Business 65, 199; Chan, K., Chan, K.C., Karolyi, G.A. (1991): The Review of Financial Studies 4, 657

Bouchaud, J.-P., Sornette, D. (1994): J. Phys. I (France) 4, 863; Sornette, D., Johansen, A., Bouchaud, J.-P. (1996): J. Phys. I (France) 6, 167

Buldyrev, S.V., Amaral, L.A.N., Havlin, S., Leschhorn, H., Maass, P., Salinger, M.A., Stanley, H.E., Stanley, M.H.R. (1997): J. Phys. I France 7, 635; Buldyrev, S.V., Leschhorn, H., Maass, P., Stanley, H.E., Stanley, M.H.R., Amaral, L.A.N., Havlin, S., Salinger, M.A. (1997): "Scaling Behavior in Economics: Empirical 
Results and Modeling of Company Growth," in Proceedings of the International School of Physics "Enrico Fermi," Course CXXXIV, edited by F. Mallamace and H. E. Stanley (IOS Press, Amsterdam)

Buldyrev, S.V., Amaral, L.A.N., Havlin, S., Leschhorn, H., Maass, P., Salinger, M.A., Stanley, H.E., Stanley, M.H.R. (1997): J. Phys. I France 7, 635

Bunde, A., Havlin, S. (1996): Fractals and Disordered Systems (2nd Edition), edited by $\mathrm{A}$. Bunde and $\mathrm{S}$. Havlin (Springer, Heidelberg)

Cizeau, P., Liu, Y., Meyer, M., Peng, C.-K. Stanley, H.E. (1997): Physica A 245, 441

Cont, R. (1997): cond-mat/9705075

Cox, J., Ross, S., Rubinstein, M. (1979): J. of Financial Economics 7, 229

Dacorogna, M.M., Muller, U.A., Nagler, R.J., Olsen, R.B., Pictet, O.V. (1993): J. International Money and Finance 12, 413

Ding, Z., Granger, C.W.J., Engle, R.F. (1983): J. Empirical Finance 1, 83

Ekman, P.D. (1992): The Journal of Futures Markets 12, 365

Engle, R.F. (1982): Econometrica 50, 987

Fama, E.-F. (1970): J. Finance 25, 383

Ghashghaie, S., Breymann, W., Peinke, J., Talkner, P., Dodge, Y. (1996): Nature 381, 767; see also Mantegna, R.N., Stanley, H.E. (1996): Nature 383, 587; (1997): Physica A 239, 255; and Arneodo A. et al. (preprint)

Granger, C.W.J., Ding, Z. (1996): J. Econometrics 73, 61

Harris, L. (1986): J. of Financial Economics 16, 99

Hausdorff, J.M., Purdon, P.L., Peng, C.-K., Ladin, Z., Wei, J.Y., Goldberger, A.L. (1996): J Appl Physiol 80, 1448-1457

Hausdorff, J.M., Peng, C.-K. (1996): Phys Rev E 54, 2154-2157

Hirabayashi, T., Takayayasu, H., Miura, H., Hamada, K. (1993): Fractals 1, 29-40

Ivanov, P.Ch., Rosenblum, M.G., Peng, C.-K., Mietus, J., Havlin, S., Stanley, H.E., Goldberger, A.L. (1996): Nature 383, 323

Ivanov, P.Ch., Rosenblum, M.G., Peng, C.-K., Mietus, J., Havlin, S., Stanley, H.E., Goldberger, A.L. (1998): Physica A 249, 587-593

Iyengar, N., Peng, C.-K., Morin, R., Goldberger, A.L., Lipsitz, L.A. (1997): Am J Physiol 271, R1078-R1084

Keitt, T., Stanley, H.E. (1998): "Dynamics of North American Breeding Bird Populations," Nature 393, 257

Keitt, T., Stanley, H.E. (preprint): "Lévy Flights and $1 / f$ Noise in High Abundance Populations"

Knobler, C.M., Robledo, A., Stanley, H.E. (1997): Statistical Mechanics in the Physical, Biological, and Social Sciences: Festschrift in Honor of Benjamin Widom on the occasion of his 70th Birthday (Elsevier, Amsterdam, 1997) [Published as a special issue of Physica A $\mathbf{2 4 4}$

Kolmogorov, A.N. (1961): Local structure of turbulence in fluid for very large Reynolds numbers. In Transl in Turbulence. Friedlander SK and Topper L, eds. (Interscience Publishers, New York), pp. 151-155

Krugman, P.R. (1996): The Self-Organizing Economy (Blackwell Publishers, Cambridge)

Levy, M., Levy, H., Solomon, S. (1994): Economics Letters 45, 103-111; Levy, M., Levy, H., Solomon, S. (1995): J. Phys. I 5, 1087-1107 
Levy, M., Persky, N., Solomon, S. (1996): Int. J. of High Speed Computing 8, 93-113; Levy, M., and Solomon, S. (1996): Int. J. Mod. Phys. C 4, 7

Liu, Y., Cizeau, P., Meyer, M., Peng, C.-K., Stanley, H.E. (1997): Physica A 245, 437

Makse, H.A., Havlin, S., Stanley, H.E. (1995): Nature 377, 608 Makse, H.A., Andrade, J., Batty, M., Havlin, S., Stanley, H.E., preprint: "Modeling Urban Growth Patterns with Correlated Percolation"

Makse, H.A., Cizeau, P., Stanley, H.E. (1997): Phys. Rev. Lett. 78, 3298

Makse, H.A., Havlin, S., King, P.R., Stanley, H.E. (1997): Nature 386, 379

Makse, H.A., Cizeau, P., Havlin, S., King, P.R., Stanley, H.E. (1998): "Spontaneous Self-Stratification Without Shaking: "Potatoes from Mashed Potatoes" [Proc. 1997 NATO ASI] in Granular Matter, edited by H. J. Herrmann (Kluwer, Dordrecht)

Makse, H.A., Cizeau, P., Stanley, H.E. (1998): Physica A 249, 391; Makse, H.A., Havlin, S., King, P.R., Stanley, H.E. (1998): Proc. Minerva Conf., Phil. Mag. 77, 1341-1351

Makse, H.A., Ball, R.C., Stanley, H.E., Warr, S. (1998): "Dynamics of Granular Self-Stratification," Phys. Rev. E 58

Mandelbrot, B.B. (1997): Fractals and Scaling in Finance: Discontinuity, Concentration, Risk (Springer-Verlag, New York)

Mandelbrot, B.B. (1963): J. Business 36, 393

Mandelbrot, B.B., van Ness, J.W. (1968): SIAM Rev. 10, 422

Mantegna, R.N., Stanley, H.E. (1995): Nature 376, 46; (1994): Phys. Rev. Lett. 73, 2946

Mantegna, R.N., Stanley, H.E. (1997): "Physics Investigation of Financial Markets," in Proceedings of the International School of Physics "Enrico Fermi," Course $C X X X I V$, edited by F. Mallamace and H. E. Stanley (IOS Press, Amsterdam, 1997); Econophysics (Cambridge University Press, under contract)

Peng, C.-K., Buldyrev, S.V., Goldberger, A.L., Havlin, S., Sciortino, F., Simons, M., Stanley, H.E. (1992): Nature 356, 168

Peng, C.-K., Mietus, J., Hausdorff, J.M., Havlin, S., Stanley, H.E., Goldberger, A.L. (1993) Phys. Rev. Lett. 70, 1343

Peng, C.-K., Buldyrev, S.V., Havlin, S., Simons, M., Stanley, H.E., Goldberger, A.L. (1994): Phys. Rev. E 49, 1684

Peng, C.-K., Havlin, S., Stanley, H.E., Goldberger, A.L. (1995): Chaos 5, 82

Peterson, I. (1996): Science News 150, 104

Peterson, I. (1997): The Jungles of Randomness (New York: Viking)

Potters, M., Cont, R., Bouchaud, J.-P. (preprint): "Financial markets as adaptative systems"

Stanley, H.E. (1971): Introduction to Phase Transitions and Critical Phenomena (Oxford, Oxford University Press)

Stanley, M.H.R., Amaral, L.A.N., Buldyrev, S.V., Havlin, S., Leschhorn, H., Maass, P., Salinger, M.A., Stanley, H.E. (1996): Nature 379, 804

Stanley, M.H.R., Amaral, L.A.N., Buldyrev, S.V., Havlin, S., Leschhorn, H., Maass, P., Salinger, M.A., Stanley, H.E. (1996): Nature 379, 804

Suki, B., Barabási, A.-L., Hantos, Z., Peták, F., Stanley, H.E. (1994): Nature 368, 615-618 
Takayayasu, H., Miura, H., Hirabayashi, T., Hamada, K. (1992): Physica A 184, 127-134

Takayasu, H., Okuyama, K. (1998): Fractals 6, 67-79

Wood, R.A., McInish, T.H., Ord, J.K. (1985): J. of Finance 40, 723

Vespignani, A., Zapperi, S. (1997): Phys. Rev. Lett. 78, 4793

Viswanathan, G.M., Afanasyev, V., Buldyrev, S.V., Murphy, E.J., Prince, P.A., Stanley, H.E. (1996): Nature 381, 413

Viswanathan, G.M., Buldyrev, S.V., Havlin, S., da Luz, M.G., Raposo, E., Stanley, H.E. (submitted) : "Optimizing Success in Random Foraging: A Physical Approach," Phys. Rev. Lett.

Zanette, D., Manrubia, S. (1997): Phys. Rev. Lett. 79, 323 